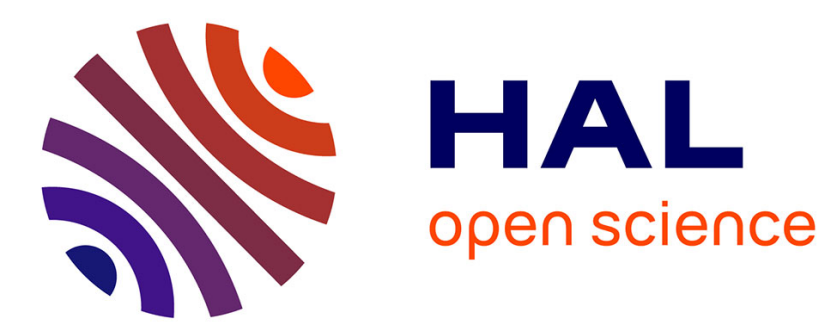

\title{
Le politique, le savant et le populaire
}

Isabelle Mayaud

\section{To cite this version:}

Isabelle Mayaud. Le politique, le savant et le populaire: L'institution d'un canon des chansons populaires de tradition nationale française au mitan du XIXe siècle. Genèses. Sciences sociales et histoire, 2019, 10.3917/gen.116.0005 . hal-02285226

\section{HAL Id: hal-02285226 \\ https://hal.science/hal-02285226}

Submitted on 12 Dec 2021

HAL is a multi-disciplinary open access archive for the deposit and dissemination of scientific research documents, whether they are published or not. The documents may come from teaching and research institutions in France or abroad, or from public or private research centers.
L'archive ouverte pluridisciplinaire HAL, est destinée au dépôt et à la diffusion de documents scientifiques de niveau recherche, publiés ou non, émanant des établissements d'enseignement et de recherche français ou étrangers, des laboratoires publics ou privés. 
Citation : Mayaud, Isabelle. «Le politique, le savant et le populaire. L'institution d'un canon des chansons populaires de tradition nationale française au mitan du XIX $^{\mathrm{e}}$ siècle », Genèses, vol. 116, no. 3, 2019, pp. 5-26.

DOI : 10.3917/gen.116.0005

Me contacter : isabelmayaud@gmail.com

Résumé : Cet article propose une revisite de "l'enquête Fortoul », une collecte de poésies populaires menée en France sous l'égide du ministère de l'Instruction publique et des Cultes, entre 1852 et 1877 . À rebours d'une lecture historiographique dominante qui considère qu'à cette date la définition d'une chanson de tradition nationale française est stabilisée, cette étude montre comment un répertoire national de référence est formé dans cette configuration historique spécifique et institué via un appareil d'État. Il s'agit ainsi de contribuer, d'une part, à une sociologie historique du politique en questionnant les fondements culturels d'un modèle d'État-nation à la française et, d'autre part, à une sociologie de l'activité scientifique et artistique par la mise au jour des mécanismes de production d'une hiérarchie de valeurs dans les domaines littéraire et musical.

\begin{abstract}
This article proposes to re-examine the "Fortoul Survey", a collection of popular poetry compiled in France under the auspices of the Minister for Public Instruction and Worship, between 1852 and 1877. Going against a dominant historiographic interpretation which considers that the definition of what constituted an authentic song in the French national tradition was stabilized by this date, this study demonstrates how a national collection of recognised songs was created within this specific historical configuration and carried out via a State body. Thus, the article aims to contribute, on the one hand, to a historical sociology of politics by questioning the cultural foundations of a French NationState model, and on the other, to a sociology of scientific and artistic activity by identifying the mechanisms producing a hierarchy of values within the fields of music and literature.
\end{abstract}

Resumen : Este artículo propone revisitar la «Enquête Fortoul», una compilación de poesía popular realizada en Francia bajo la égida del Ministerio de Instrucción Pública y de Cultos entre 1852 y 1877 . Contrariamente a una lectura historiográfica dominante que considera que en esta fecha la definición de una canción de tradición nacional francesa era ya estable, este estudio muestra cómo un repertorio nacional de referencia se formó en esta configuración histórica específica y se instituyó mediante un aparato estatal. Se trata pues de contribuir, por una parte, a una sociología histórica de la política al cuestionar los fundamentos culturales de un modelo de Estado-nación a la francesa y, por otra parte, a una sociología de la actividad científica y artística al actualizar los mecanismos de producción de una jerarquía de valores en los ámbitos literario y musical. 


\title{
Le politique, le savant et le populaire
}

L'institution d'un canon des chansons populaires de tradition nationale française au mitan du XIXe siècle

\author{
Isabelle Mayaud
}

\section{Version auteure}

Le 13 septembre 1852, Louis-Napoléon Bonaparte ordonne, par décret, la publication d'un Recueil général des poésies populaires de la France ${ }^{1}$. Débutée sous la IIe République, l'entreprise se déploie tout au long du Second Empire pour finalement s'achever sous la IIIe République : la collecte de poésies populaires de la France est officiellement interrompue en 1877. Connue sous la dénomination "d'enquête Fortoul », du nom du ministre de l'Instruction publique et des Cultes de l'époque, Hippolyte Fortoul (1811-1856), cette collecte publique est déjà bien documentée (Dubuc 1952 ; Fulcher 1980 ; Cheyronnaud 1997 ; Laborde 1998 ; Millot et al. 2005 ; Simonsen 2005 ; Berthou-Bécam et Bécam 2010 ; Campos 2013). L'épisode est aujourd'hui considéré comme l'un des moments phare de l'histoire de la patrimonialisation des chansons traditionnelles en Europe et de la fabrique concomitante des identités nationales (Laborde 1998 : 65 ; Thiesse 2001). L'entreprise est le plus souvent resituée dans une histoire longue des collectes de chansons, depuis celles réalisées par l'Académie celtique puis par les écrivains romantiques (Nerval, Sand, etc.) au cours de la première moitié du xixe siècle, jusqu'à celles qu'encouragent, à partir des années 1880, différentes sociétés et revues savantes comme la Société des traditions populaires et sa Revue des traditions populaires (Tiersot 1931 ; Bénichou 1970 ; Belmont 1975 ; id. 1986 ; id. 1995 ; Valière 2002 ; Charles-Dominique 2009 ; Fabre et Privat 2011 ; Gauthier 2013 ; CharlesDominique 2013).

Cette lecture dominante présente deux difficultés. En premier lieu, la perspective généalogiste présuppose une stabilité de l'objet - la chanson populaire traditionnelle - qu'elle contribue de façon performative à naturaliser. Il s'agit en réalité d'un exemple, classique, de reconstruction rétrospective par la mise en récit, ce que Pierre Bourdieu nomme l'illusion biographique (Bourdieu 1986). Le biais présentiste est ici patent. Dans le domaine des études musicales, plusieurs auteurs se sont attachés à penser l'enquête dans une histoire générale de la transcription des musiques populaires (Cheyronnaud 1986 ; Laborde 1998 ; Campos 2013). De façon plus large, il paraît admis que cette collecte marque une étape décisive dans l'histoire de l'appropriation savante, par « arraisonnement graphique » (Goody 1986 [1978]), de musiques non écrites. Pourtant, si on relit les premiers textes initiateurs de la collecte - le décret du 13 septembre, ainsi que le rapport rédigé par le ministre Fortoul qui a vocation à

\footnotetext{
${ }^{1}$ ANF, F17 3245 : Décret ordonnant la publication d'un Recueil général des poésies populaires de la France, 13 septembre 1852. Une version imprimée du texte est reproduite dans le Bulletin du Comité (Comité de la langue, de l'histoire et des arts de la France $1854: 22-23)$. Je remercie chaleureusement Talia Bachir-Loopuyt, Clara Biermann, Karim Hammou et Olivier Roueff pour leurs relectures critiques. Cet article doit beaucoup à la réflexion collective que nous avons engagée depuis 2013 sur les catégories musicales. Je suis également reconnaissante aux membres du comité de rédaction de Genèses pour leurs remarques constructives et stimulantes.
} 
préciser « l'esprit » du décret ${ }^{2}$ - force est de constater qu'il n'est pas question de musique. Selon la terminologie de l'époque, en 1852, tous les chants sont des poésies, mais toutes les poésies ne sont pas nécessairement chantées. La distinction est alors claire entre la poésie des chansons (les paroles) d'une part, et leur musique (l'air) d'autre part. À l'issue de cette collecte, il s'agit d'abord de parvenir à élever un vaste monument à la « gloire littéraire ${ }^{3}$ »du pays. Le caractère incontournable de la musique, et a fortiori de l'entité « chanson populaire traditionnelle ", qui semble aujourd'hui si évident dans les ouvrages qui se réfèrent à cet épisode, ne va alors pas de soi $^{4}$. La seconde difficulté que posent les lectures linéaires résulte d'un effet d'homogénéisation sociale induit par les exposés chronologiques. Les écrivains, les savants, l'appareil d'État, les associations d'érudits se trouvent fréquemment réunis en une même frise, d'une façon indifférenciée. Les études portant sur cette collecte publique présentent ainsi deux écueils significatifs : d'une part, elles occultent le processus de définition d'une « chanson populaire traditionnelle », d'autre part, elles minorent le rôle de l'appareil d'État français dans l'institution de ce répertoire.

À partir de l'examen de différentes ressources manuscrites et imprimées qui permettent de documenter le déroulement et les produits de la collecte, cet article propose une revisite de «l'enquête Fortoul ». À rebours de certaines études qui considèrent qu'à cette date, la définition d'une chanson (paroles et musique) de tradition nationale française est acquise et stabilisée, il s'agit de montrer comment un répertoire national de référence est constitué dans cette configuration historique spécifique. Dans cette perspective, la notion de « frontière » est mobilisée comme un véritable concept analytique opératoire ${ }^{5}$. Elle permet de reconsidérer cet épisode en interrogeant les modalités de production et de perpétuation d'un patrimoine populaire national ${ }^{6}$. La circonscription des contours du «populaire » devient en effet l'enjeu de discussions savantes qui concourent in fine à prescrire une définition conventionnelle inédite. Dans le sillage d'autres travaux qui ont contribué à l'historicisation du développement de canons musicaux en Occident à partir du XVIIIe siècle (Weber 1989 ; id. 1996 ; Gelbart 2007), cet article vise à mettre au jour les conditions de l'émergence d'un canon d'une chanson populaire française, le populaire se confondant ici avec le national.

Afin d'éclairer les modalités de l'institution de ce répertoire, un examen au plus près des différentes étapes de la collecte s'avère nécessaire. On peut distinguer trois grandes périodes. Un premier temps, de l'automne 1852 à l'automne 1853, correspond à une phase d'innovation au cours de laquelle le périmètre de la collecte se trouve délimité et arrêté dans

\footnotetext{
${ }^{2}$ ANF, F17 3245 : Rapport du ministre H. Fortoul au prince-président de la République française, 13 septembre 1852.

${ }^{3}$ ANF, F17 3245 : Rapport du 13 septembre 1852.

${ }^{4}$ Selon Lothaire Mabru, la notion de « musique traditionnelle » se diffuse en France à partir des années 1970 (Mabru 2007).

${ }^{5}$ Sur la « frontière » comme concept, voir Lamont et Molnár (2002) ; Jeanpierre (2010) ; Lamont, Pendergrass et Pachucki (2015).

6 À l'instar des recherches menées par Colette Guillaumin sur l'idéologie raciste : « la langue y est [ici] le "symptôme", non l'objet. Il ne s'agit pas d'une réflexion sur la langue mais d'une réflexion sur l'altérité dont le matériel est la langue » (Guillaumin 2002 [1972] : 194).
} 
un document cadre : des Instructions ${ }^{7}$. Une deuxième période constitue une phase de collecte routinisée, de l'automne 1853 à l'été 1857 . C'est le temps de la moisson au cours duquel les poésies sont rassemblées en masse. S'ouvre ensuite une ultime période, qui s'étend jusqu'en 1877, durant laquelle le projet périclite. Ma démonstration s'arrime à la temporalité de cet épisode et s'organise en trois temps. Le premier volet de l'article questionne les fondements politiques du projet. Il montre comment le Recueil des poésies populaires participe d'un dispositif de contrôle des discours qui mobilise l'ensemble de l'appareil d'État au lendemain de la révolution de 1848. Le deuxième volet présente un examen des premiers mois de la mise en oeuvre de la collecte. Il restitue le travail social de délimitation des contours discursifs du populaire par une élite savante. Le troisième volet porte enfin sur les produits de la collecte. La réflexion sur pièces permet d'appréhender certains des mécanismes qui contribuent dès lors non seulement à la production d'un canon d'une chanson populaire nationale, mais aussi à sa reproduction ad libitum.

Que ce canon résulte d'une histoire n'est, en définitive, pas surprenant. En revanche, qu'il surgisse précisément à l'occasion de cette collecte de poésies paraît plus énigmatique dans la mesure où il n'est initialement pas question de musique, et dans la mesure aussi où le recueil décrété en 1852 n'a jamais été publié. C'est à ce point que débute mon investigation.

\section{Le politique et le populaire}

Le caractère éminemment officiel de ce projet a souvent été souligné (Cheyronnaud 1986 : 12). Le décret de 1852 qui ordonne la publication du Recueil est signé par LouisNapoléon Bonaparte (1808-1873), alors président de la (IIe) République française, depuis son élection au suffrage universel le 10 décembre 1848. L'Empire est restauré et proclamé quelques semaines plus tard, le 2 décembre 1852 : Louis-Napoléon devient Napoléon III $^{8}$. Initiée par l'État français, la collecte de poésies populaires intervient alors que la situation politique est particulièrement instable. Les premiers mois de 1852 correspondent à une phase dictatoriale : l'état de siège, décrété à la suite du coup d'État de 1851, n'est officiellement levé que le 29 mars 1852. De nombreux soulèvements surviennent dans toute la France ${ }^{9}$. Sur fond de dépression agricole, le gouvernement craint de voir cette population de citoyens français des campagnes, nouvelle force politique « révélée » par la République et le suffrage universel, faire alliance avec des populations traditionnellement identifiées comme subversives. Bizarrement, «l'enquête Fortoul » a, jusqu'à présent, été très peu pensée dans la continuité de la Révolution française de 1848.

\footnotetext{
${ }^{7}$ Les Instructions d'Ampère ont fait l'objet de plusieurs éditions. Je me réfère ici à la version éditée dans le Bulletin du Comité de la langue qui compte 62 pages (Comité de la langue, de l'histoire et des arts de la France 1854 : 217-279). Le renvoi à cet imprimé se fait dans cet article sous cette forme abrégée : BCL 1854.

${ }^{8}$ Sur la IIe République, voir Agulhon (2002) ; Gribaudi et Riot-Sarcey (2009) ; Hayat (2014).

${ }^{9}$ Voir ce classement des soulèvements par département dans Aprile et Huard (2001: 7-13).
} 
En France « l'acmé de [la] production chansonnière a lieu après les journées de février 1848, et son déclin s'amorce dès les évènements de juin de la même année » (Benini 2014 : 24). La chanson envahit alors la scène publique (Agulhon 2002 [1973]). La mobilisation de chants par des insurgés dans une phase de mobilisation est historiquement tout sauf exceptionnelle (Eyerman et Jamison 1998 ; Traïni 2008). Elle représente, au xixe siècle, un vecteur de politisation comparable à la presse (Darriulat 2010 : 21). Aussi le pouvoir se montre-t-il particulièrement sensible aux effets d'une propagation non contrôlée de la culture séditieuse via les chansons et plus largement via toutes les formes spectaculaires.

Entre 1848 et 1852, une politique générale de régulation de l'expression publique orale et écrite - est déployée, qui emprunte les voies policière, judiciaire et législative. Les premières mesures concernent les salles de spectacle et les lieux de chanson. Les cafésconcerts se substituent aux goguettes (Condemi 1992). Apparus à la fin du XVIIIe siècle, ces cafés chantants vont se multiplier sous le Second Empire (Kimminich 1991 ; id. 1999). Contrairement au Caveau ou à la Lice chansonnière, il ne s'agit pas de lieux de création collective improvisée. Les textes des chansons qui y sont diffusés doivent préalablement être soumis à la censure (id. 1991). Ces transformations contribuent à normaliser un répertoire qui suppose « un lieu et une date identifiable » (Benini 2014 : 99). La littérature de colportage paye également un lourd tribut à cette politique. Le 22 janvier 1852, un ministère de la Police générale est créé, dont la direction est confiée à Charlemagne-Émile de Maupas (1818-1888). La « circulaire Maupas » du 28 juillet 1852 impose que chaque ouvrage autorisé à la vente par colportage soit frappé d'un timbre préfectoral, l'estampille. Une autre circulaire, du 12 septembre 1852, prévoit l'examen des livres par une commission du colportage. Celle-ci entre en fonction le 30 novembre 1852. Charles Nisard (1808-1890) est nommé secrétaire adjoint de la commission d'examen. Cet épisode, contemporain de «l'enquête Fortoul », est au coeur de l'article intitulé La beauté $d u$ mort, qui a fait date, publié par Michel de Certeau, Dominique Julia et Jacques Revel (Certeau, Julia et Revel 1993 [1974]). Les auteurs y exposent comment, dans un même mouvement, le pouvoir impérial sauvegarde cette littérature et la condamne en interdisant ses circuits de circulation ordinaires pour mieux l'intégrer à un autre marché des imprimés, plus luxueux et destiné aux élites. Certains des textes soumis à la commission sont en effet rassemblés puis édités par Nisard ${ }^{10}$. Comme le souligne Bernard Pudal, il n'est pas anodin que la première histoire de cette littérature de colportage soit l'œuvre d'un haut fonctionnaire chargé de la censure de ces mêmes ouvrages (Pudal 1991 : 56).

Au total, c'est l'ensemble du circuit de production de biens culturels qui se recompose au cours de cette période. S'amorce alors un reflux général de la production littéraire (Charle 1979 : 31). La littérature de colportage et les sociétés chantantes disparaissent, ce qui fait de la IIe République, comme l'observe Romain Benini, « un moment clé dans l'histoire de la

\footnotetext{
${ }^{10}$ Il publie pour la première fois, en 1854, une Histoire des livres populaires ou de la littérature de colportage : depuis l'origine de l'imprimerie jusqu'à l'établissement de la commission d'examen des livres de colportage : 30 novembre 1852. Cet ouvrage reparait augmenté, en deux volumes en 1864.
} 
chanson au XIXe siècle » (Benini 2014 : 24). Ce versant brutal des années postrévolutionnaires est aujourd'hui le mieux documenté. La politique culturelle, engagée par Louis-Napoléon sous la IIe République et développée sous l'Empire, n'est toutefois pas réductible à un strict modèle répressif.

\section{Un monument « à notre gloire littéraire » nationale}

En réalité, comme le souligne Maurice Agulhon, il s'agit bien plutôt d'une « sélection » à laquelle entend procéder le nouveau régime (Agulhon 1975 : 64). Pour preuve, Agulhon évoque précisément le Recueil des poésies populaires. Si la prohibition et la répression, en réaction aux journées de 1848, constituent les leviers privilégiés dans un premier temps par le pouvoir, la prescription d'un discours légitime constitue un autre pan de sa politique moins exploré.

Suivant les termes du rapport rédigé par le ministre Hippolyte Fortoul, le Recueil des poésies populaires est conçu, par Louis-Napoléon, comme un vaste monument à « élever à notre gloire littéraire ${ }^{11}$ ». Il s'agit de former un répertoire de référence de chants nationaux dans le sillage de ceux déjà produits par d'autres nations européennes et, par cette entreprise, de contribuer à l'unification politique du pays. L'historicité des processus d'identification culturelle et de fabrique du sentiment d'appartenance nationale a été mise en lumière par différents travaux (Anderson 1983 ; Gellner 1983 ; Hobsbawm et Ranger 1983 ; Avanza et Laferté 2005). Anne-Marie Thiesse a aussi montré combien « l'invention des nations coïncide avec une intense création de genres littéraires ou artistiques et de formes d'expression. Le retour aux origines est en fait oeuvre d'avant-garde » (Thiesse 2001 [1999] : 21). À partir de la seconde moitié du XVIIIe siècle, l'exhumation de patrimoines littéraires nationaux concourt ainsi à une «nationalisation de la culture » européenne (Löfgren 1989). Plusieurs épopées nationales fleurissent au cours de cette période : l'épopée ossianique de James Macpherson en Écosse, le Kalevala en Finlande, le Kalevipoeg en Estonie, etc. L'inspiration européenne du projet français est expressément rappelée par le ministre Fortoul lorsqu'il s'adresse aux inspecteurs de l'instruction publique afin de les encourager à collecter les poésies : «La plupart des États européens ont recueilli avec respect ces souvenirs épars des premiers efforts de leur génie national. La France seule, dont la langue est devenue européenne, négligera-t-elle ces précieuses origines d'une littérature qui fait sa gloire ${ }^{12}$ ? »

Nommé ministre au lendemain du coup d'État, Hippolyte Fortoul est chargé de l'exécution du décret. Fortoul est né à Digne le 4 août 1811 : en 1852, il a 41 ans (Massa-Gille 1995 : 533-534). À cette date, il mène de front une double activité académique et politique. L'obtention d'un doctorat ès lettres à Lyon en 1840 amorce sa carrière d'universitaire. La révolution de 1848 marque le début de sa carrière politique. Il est député des Basses-Alpes dans les rangs des conservateurs. Il est ensuite nommé ministre de la Marine puis de

\footnotetext{
${ }^{11}$ ANF, F17 3245 : Rapport du 13 septembre 1852.

12 ANF, F17 3245 : Circulaire du ministre H. Fortoul envoyée aux inspecteurs de l'Instruction primaire, novembre 1853 .
} 
l'Instruction publique et des Cultes. Fortoul vise, vraisemblablement dès 1838 , un siège à l'Académie des inscriptions et belles-lettres (Raphael et Gontard 1975 : 22). À cette fin, il publie plusieurs études sur l'art et la littérature ${ }^{13}$. «Tracer une histoire universelle de l'art »: tel est le dessein général qui préside à son œuvre (Vaisse 2000 : 145). La comparaison des productions littéraires nationales sert un discours promotionnel destiné à démontrer la supériorité de la France sur les autres pays : « de l'étude comparée des littératures, il résulte que la France, dans tout le Moyen Âge et dès ses commencements, a été, presque en tous genres, l'institutrice des nations » (Ravaisson 1856 : 4). En 1852, Fortoul compte parmi les principaux spécialistes de l'histoire littéraire nationale. Il lui revient de mettre en œuvre une enquête qui portera finalement son nom.

Monument littéraire à la gloire de la nation française, le Recueil se comprend ici dans une double logique d'affirmation nationale et de concurrence internationale. L'initiative sonne comme une réplique politique à des enjeux d'ordre politiques. Elle révèle une volonté de prescription d'un répertoire culturel légitime, ce dessein monumental ayant vocation à servir des intérêts d'unification politique nationale : autrement dit, afin de représenter cette « communauté imaginée » (Anderson 1983), « mieux vaut une douce chanson ${ }^{14} »$.

\section{Le savant et le populaire}

L'initiative intervient dans le cadre d'une politique générale qui mobilise différents agents de l'appareil d'État : des militaires, des policiers, des juristes, ou encore des savants. Décidée par le président et engagée sous l'égide du ministère de l'Instruction publique et des Cultes, la collecte est un projet éditorial. La réalisation du Recueil est, en conséquence, déléguée à l'un des services responsable des éditions imprimées au sein du ministère : la section de philologie, qui est elle-même une subdivision du Comité de la langue, de l'histoire et des arts de la France, rattaché à la division des Sciences et Lettres ${ }^{15}$. Cette section compte alors une douzaine de savants parisiens occupant des positions académiques dominantes dans les domaines de la littérature, de l'histoire littéraire et de la philologie. Conformément à l'arrêté en date du 14 septembre 1852 qui fixe pour partie l'organisation du travail des sections du Comité, la section de philologie se réunit mensuellement à partir du mois de novembre 1852. Elle rejoint par ailleurs les deux autres sections - d'histoire et d'archéologie - du Comité, le premier lundi de chaque mois. Les procès-verbaux, comptes rendus officiels des séances, sont reproduits dans le Bulletin du Comité et permettent de suivre le petit groupe au travail. En position centrale, cette section d'élite doit composer avec d'autres agents mobilisés par l'État pour l'occasion - j'y reviendrai - qui se répartissent sur tout le territoire administratif de la France. C'est à leur intention que la section entreprend de rédiger des Instructions.

\footnotetext{
${ }^{13}$ Pour un relevé de ses publications, voir Fortoul (1989).

${ }^{14}$ Extrait de la préface de 1851 de La petite fadette de George Sand (cité dans : Ponton 1977 : 62).

15 Sur l'histoire administrative de cette division, voir Antoine (1977). Sur l'histoire générale du Comité, voir Theis (1986) ; Poulot et Wrigley (1988) ; Chaline (1998) ; Leroy (2001) ; Gerson (2003).
} 
La collecte pose d'emblée la question du rassemblement des données à distance puisqu'il s'agit de recueillir les poésies « de la France ». Une définition initiale du périmètre légitime des poésies populaires circule, dès le mois de septembre 1852, via différents supports de diffusion officiels, à l'instar du Moniteur universel qui reproduit le texte du décret. Ce document est peu disert quant à la nature des pièces à retenir. Le Recueil doit comprendre « les chants religieux et guerriers ; les chants de fête, les balades ; les récits historiques, les légendes, les contes, les satires ${ }^{16} »$. Aucune indication n'est apportée quant à la langue, la date de composition ou l'auteur (ou l'absence d'auteur). Nulle mention n'est faite de la musique. En réponse à cet appel initial, plusieurs envois parviennent à la section de philologie, qui se réunit pour la première fois le 15 novembre 1852. Cette séance est l'occasion d'un premier tri et délivre un aperçu de la plurivocité des définitions des «poésies populaires » alors en circulation.

«M. Blanc, à Marseille, adresse une ancienne chanson de nourrice, en provençal, paroles et musique.

M. Lagravère, employé au ministère de l'intérieur, adresse les paroles et la musique de Louis Tilloulés ou Tillolés, ancienne chanson patoise des bateliers de la Nive et de l'Adour, à Bayonne.

M. Marcel Canat, correspondant, envoie un noël représentant la famille sainte qui cherche à loger à Châlon-sur-Saône.

M. A. Lédan, imprimeur à Morlaix, envoie un certain nombre de poésies en bas breton, manuscrites et imprimées, composées et publiées par lui, et qu'il affirme être devenues populaires dans la Bretagne. » (BCL $1854: 25)$

Cet échantillon illustre l'étendue du périmètre des " poésies populaires " tel que compris in situ. Il témoigne d'une variété de déclinaisons possibles en termes de langue (idiomes ou langue nationale), de région de provenance, d'auctorialité (connue ou non), ou encore de support de circulation (oral, manuscrit, imprimé). En outre, deux contributeurs adjoignent, de façon spontanée, la musique aux paroles.

\section{La circonscription du répertoire à collecter}

Si plusieurs documents officiels encadrent, à partir du mois de septembre 1852, le périmètre de la collecte, le principe d'Instructions destinées à préciser le corpus est également pensé, en amont, par les commanditaires ${ }^{17}$. La diffusion d'Instructions constitue alors une procédure routinisée pour le Comité. Avec le questionnaire, il s'agit de l'un des principaux instruments de collecte de données à distance depuis l'époque moderne (Blanckaert 1996 ; Collini et Vannoni 2005). Largement commentées par l'historiographie, les Instructions gagnent à être étudiées sous l'angle du processus qui préside à leur élaboration à partir du mois de novembre 1852. La commission en charge de leur rédaction se compose initialement

\footnotetext{
${ }^{16}$ ANF, F17 3245 : Décret du 13 septembre 1852.

${ }^{17}$ On trouve trace de ce projet d'Instructions dans la séance générale d'ouverture du Comité du 8 novembre 1852.
} 
de trois membres de la section de philologie, tous rattachés à l'Académie des inscriptions et belles lettres : Jean-Jacques Ampère (1800-1864), Alexis Paulin Pâris (1800-1881) et Charles Magnin (1793-1862). Ampère évince rapidement Pâris et Magnin : il est présenté à l'automne 1853 comme l'unique auteur de ce que l'on désigne communément comme « les Instructions d'Ampère ». Ce document fait néanmoins l'objet de discussions collectives en séance. L'examen de ces dernières permet de saisir un double mouvement, d'une part, de resserrement du périmètre à certaines paroles des poésies et, d'autre part, d'élargissement à leur musique.

La délimitation du corpus à certains textes constitue un enjeu majeur au sein de la section de philologie. Les débats qui animent ce groupe savant sont d'abord ceux de spécialistes d'histoire littéraire. Les questions de classification générique sont, par exemple, soulevées lors de la séance du 14 février 1852 : «La section examine, en premier lieu, si le recueil se composera uniquement de chants, ou s'il comprendra également les poésies chantées et les poésies récitées »(BCL 1854 : 99). Le décret spécifiant que les récits historiques doivent être inclus, la section ne peut exclure les poésies récitées. De façon pragmatique et afin de limiter le périmètre de la collecte, la section introduit alors la prévalence de deux variables, déterminantes à ce stade du processus. Elle retient de préférence, d'une part, « les poésies anonymes composées par ou pour le peuple, et qui ont été récitées sur les places publiques » (BCL 1854 : 99) et, d'autre part, les poésies orales « conservées par le chant et la narration orale, qui se sont transmises par la tradition de génération en génération » (ibid.). Les clauses d'anonymat et d'oralité sont dès lors déterminantes dans la constitution du corpus.

L'élargissement à la musique intervient dans un second temps. La question des airs fait une première irruption discrète lors de la séance générale du Comité, le 7 mars 1853 : «M. Vincent demande si la section de philologie a pris quelque résolution au sujet de la musique des chants populaires. M. Guigniaut répond que la section considère la musique des chants populaires comme un élément essentiel qui ne saurait être séparé des poésies » (BCL 1854 : 148). Alexandre-Joseph Vincent (1797-1868) est l'une des principales personnalités qui s'adonnent, à partir des années 1830 , à des travaux d'archéologie musicale ${ }^{18}$. Il est membre de la section d'archéologie du Comité, au sein de laquelle les recherches musicales occupent une place importante ${ }^{19}$. Au printemps 1853, Vincent prend en charge la rédaction de la partie musicale des Instructions dont il présente une première version lors de la séance du 25 avril 1853 de la section d'archéologie. L'académicien Joseph-Victor Le Clerc (1789-1865) propose quant à lui un certain nombre de correctifs à apporter aux Instructions lors de la séance du 11 avril réunissant la section de philologie. Il milite en faveur du maintien des poésies manuscrites dans le répertoire légitime à investir. Dans cette perspective, il fonde son argument sur deux exemples puisés dans l'histoire littéraire du XIIIe siècle, qui attestent que les chansons de geste ne sont pas seulement récitées mais aussi chantées. Gage de la popularité d'une pièce, la musique vaut ici pour argument justifiant son inclusion dans le

\footnotetext{
${ }^{18}$ On se reportera aux travaux de Sophie-Anne Leterrier sur l'archéologie musicale (Leterrier 2005 ; id. 2006).

${ }^{19}$ Des Instructions sur la musique sont rédigées par l'archéologue de la musique Auguste Bottée de Toulmon (1797-1850) et publiées par le ministère en 1839.
} 
corpus. Dès lors, elle s'impose comme objet d'investigation légitime de cette enquête. La section de philologie se réunit une dernière fois le 27 juillet, l'élaboration collective des Instructions s'achève alors que débute la pause estivale. Elles comportent une soixantaine de pages, dont deux spécifiquement dédiées à la musique comprise au sens limité des airs des chansons (BCL 1854 : 226-227).

\section{Le canon des « chansons populaires » en 1853}

Les contours des poésies populaires sont précisément fixés et illustrés de nombreux exemples dans les Instructions qui stipulent un premier cadrage d'ordre générique au double sens de délimitation des genres littéraires visés par la collecte et des caractères génériquement partagés. Différents critères sont ainsi arrêtés : la chronologie, la géographie, la langue, le support de conservation, le type de poésie, l'auctorialité, le genre littéraire (Tableau 1). Plusieurs genres considérés au cours de l'année sont exclus du périmètre légitime d'investigation, à l'instar des chansons de geste - c'est-à-dire des poésies épiques du Moyen Âge transmises par le chant ou la récitation orale, le parangon étant La chanson de Rolandou encore des poésies lyriques des troubadours et des trouvères, au motif qu'elles sont « un produit de l'art» (BCL $1854: 225)$.

Tableau 1. Les caractéristiques des poésies populaires suivant les Instructions

\begin{tabular}{|l|l|}
\hline Chronologie & Périmètre \\
\hline Géographie & poésies antérieures au XIXe siècle \\
\hline Langue & $\begin{array}{l}\text { France dans ses limites politiques contemporaines (dont les } \\
\text { territoires nouvellement conquis ou annexés) ; France dans ses } \\
\text { limites antérieures (tout territoire occupé par des Français) } \\
\text { repérables à partir des survivances linguistiques }\end{array}$ \\
\hline Support de conservation & $\begin{array}{l}\text { français ; allemand ; flamand ; bas-breton ; italien ; catalan ; } \\
\text { basque ; latin ; moitié français, moitié latin ; français du Moyen } \\
\text { Âge ; créole }\end{array}$ \\
\hline Type de poésie & $\begin{array}{l}\text { primeur aux poésies chantées } \\
\text { poésies récitées secondaires }\end{array}$ \\
\hline Auctorialité & $\begin{array}{l}\text { primeur aux Compositions anonymes nées par le peuple } \\
\text { Compositions avec auteurs adoptées par le peuple exclusion des } \\
\text { Compositions pour le peuple }\end{array}$ \\
\hline Genre & $\begin{array}{l}\text { exclusion des chansons de geste, chansons des troubadours et } \\
\text { des trouvères }\end{array}$ \\
\hline
\end{tabular}

Les Instructions repèrent également un certain nombre de caractères qualitatifs distinctifs. Leur addition esquisse, de façon implicite, les contours d'une esthétique populaire. L'analyse du lexique employé dans ce document révèle une omniprésence de la notion d'antiquité. On trouve une multitude d'occurrences des termes suivants : « ancien », « antique », « antérieur », « tradition », « vestige », « trace », « souvenir », « débris », « primitif ». L'assignation systématique du répertoire des poésies populaires à une primitivité renvoie cette production à des temps immémoriaux. Les Instructions mobilisent ensuite un 
certain nombre d'adjectifs destinés à qualifier l'esthétique propre aux poésies populaires. Une définition qualitative s'organise ici autour de couples oppositionnels qui convoquent l'attirail disponible d'adjectifs ordinairement utilisés pour désigner et déprécier l'étrangeté (Tableau 2).

Tableau 2. Qualifier les poésies populaires

\begin{tabular}{|l|l|}
\hline \multicolumn{1}{|c|}{ Poésies populaires } & \multicolumn{1}{c|}{ Poésies littéraires } \\
\hline Naïf & Savant \\
\hline Gracieux & Mondain \\
\hline Burlesque & Sérieux \\
\hline Grossier & Fin \\
\hline Enjoué & Sérieux \\
\hline Simple & Complexe \\
\hline Involontaire & Intentionnel \\
\hline Spontané & Créé \\
\hline Bizarre & Normal \\
\hline Rustique & Raffiné \\
\hline
\end{tabular}

Dans les Instructions, la musique survient assez rapidement, à la dixième page. Les consignes établies par Vincent valent principalement pour les airs transmis par la voie orale (Tableau 3).

Tableau 3. Les caractéristiques de la musique populaire suivant les Instructions

\begin{tabular}{|l|}
\hline \multicolumn{1}{|c|}{ Musique populaire } \\
\hline Mesure et rythme irrégulier \\
\hline Air peut finir autrement que sur la tonique, sur la dominante par exemple \\
\hline $\begin{array}{l}\text { Air peut ne pas avoir de note sensible : le degré immédiatement inférieur à la tonique ne } \\
\text { diffère pas d'un demi-ton mais d'un ton plein }\end{array}$ \\
\hline
\end{tabular}

Outre la phrase mélodique simple, Vincent, qui bénéficie d'une solide formation de mathématicien, identifie trois variables principales à partir desquelles il dresse un portrait strictement relationnel des caractères mélodico-harmoniques de la musique populaire. Celle-ci est définie par ses manques et par ses absences, le référent étant le système tonal occidental. Par exemple, les airs populaires ne se terminent pas nécessairement sur la tonique. La définition de la musique populaire se comprend ici en lien avec le développement contemporain de l'archéologie musicale dont Vincent constitue l'une des figures de proue. Les règles de l'harmonie propres à cette musique sont ainsi forgées, par opposition avec la tonalité moderne et aussi par analogie avec la modalité ancienne spécifique aux chants d'église.

L'intérêt nourri par ce domaine savant pour les questions touchant à la préhistoire de l'art musical constitue l'un des ressorts favorisant le dialogue avec les spécialistes de l'histoire littéraire. La philologie intervient au point de carrefour et alimente une réflexion autour de la notion de spécimen primitif. Les préoccupations de Vincent recoupent, par exemple, celles exprimées par le secrétaire du Comité Paul-Arthur Nouail de la Villegille 
(1803-1882), cheville ouvrière de ce groupe, qui relaie systématiquement les vues de la section de philologie. Celle-ci accorde une attention particulière aux variantes des airs :

«Quand des chansons ont été composées sur un air plus ancien, en remontant aux paroles qui ont donné à l'air le nom sous lequel il est connu, on a grande chance de rencontrer un chant populaire, qui souvent a donné à l'air sa popularité » (BCL 1854 : 228).

La quête des origines suppose, pour les uns comme pour les autres, une investigation de type généalogique. À la croisée de l'histoire littéraire et de l'archéologie musicale, émerge ainsi une réflexion autour des critères de définition d'une chanson (paroles et musique) populaire que l'on assigne désormais à un passé ancien (avant le XIXe siècle) et à une modalité de circulation (la tradition). Au terme de ce tracé savant des frontières du populaire, un corpus de chansons populaires émerge, distinct des poésies littéraires et positionné dans un en-deçà de l'histoire littéraire. Dans l'intervalle, ce répertoire a subi diverses opérations qui participent d'un « processus de primitivisation » (Mayaud 2018) : issu de la tradition, transmis par la voie orale, ce répertoire est également anonymisé.

Ainsi constitué dans un petit espace savant parisien élitiste, un premier canon de la chanson populaire est fixé en 1853. L'observation de la section de philologie au travail révèle combien la musicalisation du répertoire des poésies populaires correspond d'abord à une éviction des poésies populaires de l'histoire littéraire nationale et à leur inscription concomitante dans le hors-temps de la mémoire nationale, à savoir l'éternité du peuple français. Au fil des discussions savantes, l'objet de la collecte paraît ainsi s’être déplacé.

\section{Une chanson populaire ad libitum}

$\mathrm{Au}$ terme de cette phase d'innovation, les Instructions sont diffusées dans toute la France. À partir de l'automne 1853, les poésies affluent et la section de philologie entreprend le travail de tri systématique et routinisé pour lequel elle a reçu mandat. L'essentiel de la collecte se déroule entre 1853 et 1857 . Au cours de cette période les séances de dépouillement des correspondances se succèdent régulièrement. Certaines poésies sont acceptées, d'autres refusées (Encadré).

\section{Les pièces de « l'enquête Fortoul "}

Entre le mois de novembre 1852 et le mois de juillet 1857 , la section de philologie se réunit à quarante reprises. Les pièces admises par la section sont consultables à la Bibliothèque nationale de France. Véritable « nécropole » (Gaidoz 1906 : 187), le département des manuscrits de la BNF renferme un Recueil des admis répartis en six volumes sur 3251 feuillets. Un premier volume daté du 27 février 1877 compte 645 feuillets (BNF, N.A.F. 3338) ; le deuxième, daté du 27 février 1877, 392 feuillets (BNF, N.A.F. 3339) ; le troisième, daté du 28 février 1877, 552 feuillets (BNF, N.A.F. 3340) ; le quatrième, daté du 1er mars 1877, 538 feuillets (BNF, N.A.F. 3341) ; le cinquième, daté du 27 février 1877, 585 feuillets (BNF, N.A.F. 3342) ; et le sixième, daté du 27 février 1877, 539 feuillets (BNF, N.A.F. 3343). Les pièces « refusées » sont quant à elles, pour la plupart, remisées dans deux cartons aux archives nationales (ANF, F17 3245 et F17 3246). Produits et productrices d'un savoir 
original, les différentes opérations de tri ont façonné les contours du corpus aujourd'hui accessible.

Finalement, le projet commencé en 1852 « échoua puisque la publication ainsi prévue ne se fit jamais » (Cheyronnaud 1986 : 26). La faiblesse du financement, le recul de la philologie au sein du Comité et l'absence de volonté politique, paraissent des variables explicatives de la faillite de ce projet éditorial. En 1876, la décision est finalement prise, suivant les recommandations émises dans un rapport remis par l'homme de lettres Charles Marty-Laveaux (1823-1899), d'abandonner la collecte. En dépit de son inaboutissement, cette collecte a durablement contribué à l'institution d'un canon des chansons populaires de tradition française. Ce dernier volet révèle certains des mécanismes sociaux à l'œuvre qui concourent à la production et à la reproduction de ce répertoire.

\section{Un pouvoir stratifié d'imposition de la norme du populaire}

L'autorité des Instructions est en premier lieu à rapporter à la puissance de l'appareil d'État qui assure leur diffusion. Le dispositif de collecte de données s'organise en effet d'emblée suivant une logique de division et de hiérarchisation du travail à l'échelle de l'ensemble du territoire métropolitain. Un organe central - la section de philologie - est en charge du stockage et du traitement des données que lui fournit un réseau régionalisé d'informateurs. La part des académiciens, membres de l'Institut, au sein de cette section de philologie est écrasante (9 sur 12). Il s'agit d'une élite sociale qui peut compter ici sur le concours de deux canaux principaux préexistants, directement sollicités par les pouvoirs publics : les correspondants ordinaires du Comité d'une part, les fonctionnaires de l'Instruction publique et des Cultes d'autre part. En 1854, des agents de l'armée sont en outre mobilisés, de façon marginale ${ }^{20}$. Ressources ordinaires du Comité, les correspondants locaux sont, en 1852, tous nommés par arrêté ministériel et au nombre de deux cents - ce chiffre étant fixé par un arrêté du 7 avril 1851. Ce sont des notables qui résident en province et qui se répartissent sur l'ensemble du territoire national. Dans le cadre de ce projet, le ministre sollicite également des ressources extraordinaires : des fonctionnaires du ministère de 1'Instruction publique et des Cultes. Ce second réseau de collecteurs se déploie suivant une logique hiérarchique. Les recteurs sont en charge de la coordination des recherches des inspecteurs, qui sont eux-mêmes invités à évaluer le travail des instituteurs lors de leurs tournées d'inspection. Ces agents sont donc mobilisés, dans le cadre de "l'enquête Fortoul », à la marge de leur activité principale, sur la base du volontariat. Ils assurent un quadrillage administratif du territoire à la fois permanent et temporaire.

À partir des informations rassemblées par le couple Berthou-Bécam et Bécam à l'échelle de la Bretagne, il est possible d'esquisser une sociologie des collecteurs en lien avec la section (Berthou-Bécam et Bécam 2010). L'examen atteste d'une forte hétérogénéité sociale au sein du groupe de trente-cinq individus - tous des hommes - identifiés dans cette

\footnotetext{
${ }^{20}$ ANF, F17 2830/1. Lettre du maréchal de France, Ministre secrétaire d'État de la guerre au ministre Fortoul, Paris, 21 février 1854
} 
étude. Au plus haut de l'échelle sociale se trouve un rentier, Théodore Hersart de La Villemarqué (1815-1895), et au plus bas un instituteur officiant à Carhaix. Entre ces deux pôles, la population se distribue entre : 1) des enseignants, des savants et des conservateurs (des archives et des bibliothèques), membres du Comité pour la plupart ; 2) des professions libérales (magistrat, médecin, journaliste, imprimeur), les premiers étant souvent membres du Comité, les autres non ; 3) des instituteurs, des inspecteurs de l'instruction primaire ; 4) on trouve enfin un ancien militaire, un employé de l'administration publique et un compositeur de musique. Cette mobilisation est donc exceptionnelle de par son ampleur spatiale l'ensemble du territoire métropolitain - et sociale - tous les échelons de la pyramide sociale sont représentés. Le dispositif dans son ensemble suppose en réalité une division du travail de collecte et de tri qui renvoie à une stratification sociale des maîtres d'œuvre.

Le mandat, l'autorité et le moment de l'intervention au sein du dispositif sont, en outre, indexés à la position sociale des contributeurs. Les instituteurs sont, par exemple, encouragés à « interroger les souvenirs des populations agricoles, où les vieilles traditions se perpétuent si facilement ${ }^{21} \gg$. Le pouvoir de discrimination est également fonction de la position dans la hiérarchie. L'examen des nombreux cahiers produits par les instituteurs témoigne d'un contrôle exercé par les recteurs et les inspecteurs qui s'assurent du bon respect des Instructions. La langue dominante des chansons peut ici être prise pour preuve de la censure et de l'autocensure à l'œuvre. L'envoi de M. Pineau, instituteur communal à Liré (arrondissement de Beaupréau) ne comporte que des pièces en langue française ${ }^{22}$. Si les Instructions invitent à ne pas exclure les idiomes et les patois, elles enjoignent aussi les collecteurs à fournir systématiquement une traduction en français des chansons collectées : « Des traductions littérales seront mises en regard du texte original des poésies composées dans ces différents idiomes »(BCL 1854 : 219). Les illustrations fournies dans ce document prescripteur sont d'ailleurs majoritairement en français. L'horizon de référence est le monolinguisme promu par un État-nation français en construction. Cet épisode doit se comprendre dans le prolongement d'une politique de la langue initiée au lendemain de 1789 (Certeau, Julia et Revel 2002 [1975] ; Higonnet 1980). Il participe d'une dynamique de francisation qui rencontre toujours au mitan du XIXe siècle de nombreuses résistances (Niel 2007). À une logique d'éradication défendue notamment par l'abbé Grégoire (1750-1831), se substitue ici une autre logique d'intégration des particularismes linguistiques. L'analyse des centaines de feuillets manuscrits collectés entre 1852 et 1877 témoigne de ce point de vue de la réussite du projet. Les pièces en patois sont relativement rares dans ce corpus eu égard aux parlers alors en usage (Vigier 1979). Les Instructions, dont des dizaines d'agents de l'État assurent la diffusion, contribuent en ce sens à une homogénéisation du répertoire et à une imposition connexe du français comme langue universelle à l'échelle du territoire français.

\footnotetext{
${ }^{21}$ ANF, F17 3245 : Circulaire du ministre envoyée aux inspecteurs de l'instruction primaire, novembre 1853.

${ }^{22}$ BNF, N.A.F. 3342 , p. 11 recto à 18 verso.
} 
L'examen des centaines de feuillets conservés à la BNF permet, plus largement, de mesurer le degré d'incorporation de la grille d'enquête initialement fixée dans les Instructions et de s'assurer, in fine, du pouvoir de prescription de cet instrument. L'étude d'un échantillon de spécimens révèle d'abord une conception relativement flottante de la musique populaire. Dans de nombreux cas, les airs transcrits présentent certains des caractères mélodicoharmoniques stipulés par Vincent, mais pas nécessairement tous. S'agissant du choix de l'échelle, tonale ou modale, plusieurs chansons sont ambivalentes. On trouve ainsi des pièces construites sur des échelles modales. Par exemple, La chanson des cordonniers, dont l'air est noté suivant un système ancien, ne comporte pas de sensible ${ }^{23}$. Mais on trouve aussi des pièces qui peuvent très bien s'harmoniser de manière tonale. Le petit écolier recueilli par l'instituteur Pineau présente un air simple : une même mélodie, des lignes conjointes, un rythme régulier ${ }^{24}$. C'est un cantique régulier, tonal, en $d o$ majeur, qui ne correspond donc pas au critère énoncé par Vincent. Tout se passe ici comme si ces agents tentaient de se conformer aux codes et aux cadres de la modernité musicale sans y parvenir tout à fait. Nombre de collecteurs emploient, par exemple, les barres de mesure. Ils prennent néanmoins des libertés avec ce cadre initial et accommodent une organisation régulière du son en introduisant des irrégularités. Saint Vincent, patron des vignerons constitue une pièce exemplaire de ces petits écarts ${ }^{25}$. La rareté des textes d'accompagnement destinés à caractériser la musique et, au-delà, les libertés prises par les collecteurs avec les consignes diffusées dans les Instructions traduisent un défaut de formation musicale largement partagé. D'un strict point de vue quantitatif, le bilan de la collecte des airs est d'ailleurs mitigé. D'après le décompte établi par le secrétaire du Comité La Villegille en 1857 à partir de l'examen d'un échantillon de 696 morceaux, la musique figure dans $30 \%$ des cas. Le relevé réalisé à l'échelle de la Bretagne par le couple Berthou-Bécam et Bécam confirme ces proportions : la musique est présente dans $28,5 \%$ des cas. On peut ainsi estimer la part de la musique à trois mélodies notées pour dix poésies rassemblées.

L'analyse musicale apparaît en définitive accessoire dans le choix des morceaux. Plutôt qu'aux propriétés sonores, les contributeurs recourent régulièrement à différentes propriétés sociales afin d'opérer leur tri. Parmi tous les critères mis en avant au fil des feuillets, deux grands types de variables paraissent déterminants : l'anhistoricité d'une part, la provenance sociologique d'autre part. Plusieurs indications renvoient ainsi à un en-deçà du temps historique : «Cette ronde remonte à un temps très reculé mais qu'on ne peut préciser. C'est un chant tout particulier à la Gascogne ${ }^{26}$ », note un contributeur. Le caractère immémorial d'une pièce est garant de sa popularité. Dans cette perspective, les montagnes apparaissent comme un conservatoire de prédilection, dernier lieu préservé des influences étrangères, et dans lequel survit une variété de spécimens. La provenance sociologique constitue le deuxième critère principal de sélection in situ. L'accent mis sur les usages

\footnotetext{
${ }^{23}$ BNF, N.A.F. 3342, p. 95.

${ }^{24}$ BNF, N.A.F. 3342, p. 15.

${ }^{25}$ BNF, N.A.F. 3342, p. 100.

${ }^{26}$ BNF, N.A.F. 3340, p. 29.
} 
collectifs suppose admis qu'une poésie populaire est une poésie chantée par le peuple, soit une fraction spécifique de la population aux contours instables : « Je ne connais point de chanson plus populaire que celle-ci. C'est par excellence la chanson du paysan ${ }^{27}$ », affirme un autre contributeur. Le peuple s'incarne préférentiellement dans certains groupes sociaux - les enfants, les paysans, les villageois - choisis et circonscrits à partir de la mobilisation de variables hétéroclites : la génération, l'activité économique, le lieu de résidence, le sexe. Les usages individuels introduisent, quant à eux, à la rhétorique du dernier vestige. Les collecteurs fournissent souvent une information détaillée concernant l'origine sociale du chanteur ou de la chanteuse et esquissent le portrait de ces voix de la tradition. Celles-ci appartiennent à deux grands groupes sociaux : les personnes âgées et les femmes. Une poésie populaire transmise par la tradition est un spécimen rare, dont seuls quelques anciens et anciennes conservent le souvenir : « Ce chant airs et paroles m'a été communiqué par un vieillard - aujourd'hui centenaire de ma paroisse ${ }^{28}$ ", écrit ainsi un collecteur. La qualification esthétique du populaire passe enfin, dans les textes d'accompagnement fournis par les collecteurs tout comme dans les Instructions, par la mobilisation d'un lexique spécifique. De ce point de vue la définition du populaire apparaît plus pauvre. « Le chant ci-dessous paraît être Berrichon ; la poésie en est simple et naïve et la mélodie très douce et bien appropriée ${ }^{29}$ », note un collecteur. Pour l'essentiel, le style populaire est réductible à trois traits - le naïf, le simple et le rustique - qui renvoient à un caractère primitif. L'examen du corpus de chansons collectées atteste ainsi d'une popularisation d'une sélection limitée de critères prédéfinis dans les Instructions. Il témoigne également de l'efficacité du dispositif sur pièces. Le guide d'enquête apparaît ici bien suivi. Le pouvoir d'imposition de la norme du populaire apparaît en outre d'autant plus important que le contributeur à la collecte occupe une position élevée dans la pyramide sociale. En bout de chaîne se retrouvent finalement les savants réunis au sein de la section de philologie. L'examen des procès-verbaux des séances, ainsi que les pièces collectées, rassemblées à la BNF et aux ANF, permet de relever les principaux motifs de sélection invoqués par les savants. Conformément aux Instructions, la facture littéraire des poésies représente une variable exclusive importante. Un noël bressan intitulé La bolia aveulia (La jeune fille aveugle) est annoté au crayon « littéraire ${ }^{30}$ » et remisé aux archives. Toute une série de pièces envoyées par l'inspecteur des écoles de l'arrondissement de Dijon sont aussi jugées « littéraires » par Charles-Augustin Sainte-Beuve (1804-1869). Le mal d'amour $^{31}$, Lubin et Collette ${ }^{32}$, Le cocher amoureux ${ }^{33}$, La mère Jeanne ${ }^{34}$, etc., sont ainsi exclues. La section confirme un résultat important, produit du processus de conception des Instructions, puisqu'elle conforte le couple oppositionnel articulant le «populaire »vs le « littéraire ». L'institution d'un canon d'une chanson populaire de tradition nationale française passe donc par un appareil d'État qui s'impose ici comme un vecteur puissant d'imposition d'une norme. Celle-ci circule d'autant mieux entre les différentes strates sociales qu'elle n'est

\footnotetext{
${ }^{27}$ BNF, N.A.F. 3341 , p. 302.

${ }^{28}$ BNF, N.A.F. 3338, p. 88-89.

${ }^{29}$ BNF, N.A.F. 3340, p. 141.

${ }^{30}$ BNF, N.A.F. 3338, p. 251.

${ }^{31}$ BNF, N.A.F. 3342, p. 161.

${ }^{32}$ BNF, N.A.F. 3342, p. 161 verso.

${ }^{33}$ BNF, N.A.F. 3342, p. 164.

${ }^{34}$ BNF, N.A.F. 3342, p. 169.
} 
pas seulement le produit d'une réflexion savante dominante mais qu'elle est d'une certaine façon co-produite, de façon certes bridée, par des agents situés en position intermédiaire sur l'échelle sociale. Le modèle de domination déployé par l'État-nation français repose sur une logique d'intégration culturelle, à la fois sociale et spatiale.

En dépit, voire grâce à son inachèvement, " l'enquête Fortoul » est une réussite politique. L'absence de publication ainsi que la légende noire qui entoure cet épisode sous la IIIe République, à l'instar de nombre d'initiatives portées par le régime de Napoléon III, a en effet favorisé le développement d'une certaine amnésie collective ${ }^{35}$. Cet article invite à une réévaluation de l'importance de cette collecte. À rebours d'une lecture historiographique dominante, le développement qui précède montre comment la notion de patrimoine hérité acquiert toute sa pertinence à l'issue de cette enquête et non pas à ses débuts. À une conception étendue des «poésies populaires », qui ne sont pas nécessairement chantées, se substitue une conception limitée des « chansons populaires ». Cette requalification procède de différentes opérations savantes successives qui aboutissent à une éviction de ce répertoire spécifique de l'histoire littéraire et à son inscription au seul registre de la mémoire nationale. Le populaire se forge ici par opposition à l'art littéraire. Il se définit aussi par rapport à un autre populaire, commercial et urbain, déjà documenté pour cette période (Leterrier 1999). La revisite de cette collecte de poésies populaires engagée en 1852 paraît ainsi féconde à plusieurs titres. Elle révèle comment un répertoire national de référence est constitué dans cette configuration historique spécifique et institué via un appareil d'État.

Partant, l'examen de ce cas paraît susceptible de venir nourrir des réflexions dans plusieurs domaines de la connaissance : la sociologie de la culture, la sociologie des sciences et la sociologie historique du politique. Dans le sillage des nombreux travaux qui se sont attachés à dénaturaliser les hiérarchies culturelles, cette recherche invite en premier lieu à repenser les conditions sociales de l'émergence d'un canon esthétique. L'examen de cet épisode conforte notamment la thèse développée par Matthew Gelbart en confirmant, à l'échelle de la France, le caractère factice de la correspondance entre modalité et musique populaire (Gelbart 2007). En deuxième lieu, cette étude de cas informe l'histoire des relations entre le savant et le politique, d'une part (Weber 2002 [1959]), et le savant et le populaire, d'autre part (Grignon et Passeron 2015 [1989]). Les Instructions, ainsi que l'appareil d'État qui assure leur large circulation, participent pleinement d'un dispositif de production de connaissance dans lequel les savants occupent une place à la fois centrale et subordonnée. L'analyse des modalités de la collecte témoigne aussi d'une relation complexe entre le savant et le populaire. Un modèle de domination verticale, conforme à celui discuté par Grignon et Passeron, est clairement identifiable. En ce sens, cet épisode s'inscrit dans une histoire longue de la définition par les savants de la culture légitime. Il ne peut toutefois s’y réduire. En effet,

\footnotetext{
${ }^{35}$ Sur la légende noire relative à cette collecte : Mayaud (2018 : 143-144). Plus largement, sur la légende noire concernant la politique culturelle menée sous le Second Empire, voir Yon (2012 [2004]).
} 
le dispositif repose également sur une logique inclusive et suppose l'implication d'une population socialement et spatialement hétérogène. De cette collecte, il résulte tout à la fois une définition du populaire de spécialistes qui se perpétue dans le domaine savant «par le haut » mais aussi une définition produite depuis le terrain qui se popularise «par le bas ». En troisième lieu, l'étude de cet épisode paraît à même de contribuer à une sociologie historique du politique en documentant les fondements d'un modèle d'État-nation à la française. Comme l'observe Jacques Cheyronnaud, tout le dispositif de collecte concourt ici à la production d'une définition provinciale de la poésie populaire (Cheyronnaud 1986 : 72). Les modalités pratiques de la réalisation de la collecte sur le terrain contribuent autant, sinon de ce cas de collecte publique inachevée - ce qui est en définitive courant dans l'histoire des enquêtes collectives (Amiotte-Suchet et al. 2016 ; Laferté, Pasquali et Renahy 2018) - invite aussi à interroger ce que recouvre l'idée de « réussite » dans les mondes politique et scientifique. plus, que les Instructions, à la circonscription du répertoire de référence. Le rassemblement des poésies s'opère à l'échelle d'un territoire national administré et quadrillé par un appareil d'État. Le cas français apparaît en ce sens singulier. Il se distingue des autres États européens par une implication de l'appareil d'État, et non pas seulement des élites, dans la nationalisation de l'identité politique et la civilisation des esprits. Dans ce cas spécifique, l'État et la nation se construisent de concert (Linz 1997 [1993]). La multiplication des « chants populaires de » la Provence, la Bretagne, etc., édités à partir des années 1850, produits éditoriaux dérivés de " l'enquête Fortoul », constitue un bon indice de cette fixation à l'œuvre des territoires de la tradition qui suppose et produit en retour une analogie entre chant provincial et chant national ${ }^{36}$. La collecte entérine ainsi une régionalisation du répertoire des chansons populaires de France. Par toutes ces voies, cet épisode contribue au développement d'un modèle durable d'union nationale articulée aux petites patries. Il s'agit là d'une voie originale et conjoncturelle de fondation de l'unité politique qui passe par une unité culturelle plurivoque. Bien étudiée pour la IIIe République (Muel-Dreyfus 1983 ; Thiesse 1997), cette morphologie originale d'unité culturelle à la française s'esquisse ici. Elle est promise à une longue postérité (Weber 2000 ; Müller et Weber 2003 ; Christophe et al. 2009). Le succès de cette initiative politique est donc double : l'enquête produit un populaire et pose les jalons en vue de sa reproduction ad libitum. Comme le souligne le folkloriste Henri Gaidoz au début du xxe siècle, "l'impulsion avait été donnée pour les recueils de chansons populaires 》 (Gaidoz 1906 : 188). Finalement, l'examen de ce cas de collecte publique inachevée - ce qui est en définitive courant dans l'histoire des enquêtes collectives (Amiotte-Suchet et al. 2016 ; Laferté, Pasquali et Renahy 2018) - invite aussi à interroger ce que recouvre l'idée de « réussite » dans les mondes politique et scientifique.

\footnotetext{
${ }^{36}$ Sur l'essor de ces éditions, voir Mayaud (2018 : 292-299).
} 


\section{Bibliographie}

Agulhon, Maurice. 1975. « Le problème de la culture populaire en France autour de 1848 », Romantisme, no $9:$ 50-64.

Agulhon, Maurice. 2002 [1973]. 1848 ou l'apprentissage de la République : 1848-1852. Paris, Seuil.

Amiotte-Suchet, Laurent, Gilles Laferté, Christine Laurière et Nicolas Renahy. 2016. «Enquêtes collectives », Ethnographiques.org [en ligne], no 32. URL: http://www.ethnographiques.org/2016/Amiotte-Suchet-Laferte-Lauriere-Renahy (consulté le 8 août 2017).

Anderson, Benedict. 1983. Imagined Communities: Reflections on the Origin and Spread of Nationalism. Londres, Verso.

Antoine, Marie-Élisabeth. 1977. « La division des Sciences et Lettres du ministère de l'Instruction publique ", Bulletin de la section d'histoire moderne et contemporaine du Comité des travaux historiques, fasc. 10.

Aprile, Sylvie et Raymond Huard. 2001. " Le coup d'État du 2 décembre 1851. Bibliographie », Revue d'histoire $d u$ xixe siècle [en ligne], no 22. URL : http :// rh19.revues.org/252 (consulté le 10 janvier 2015).

Avanza, Martina et Gilles Laferté. 2005. "Dépasser la "construction des identités" ? Identification, image sociale, appartenance », Genèses, no 61 : 134-152.

Belmont, Nicole. 1975. "L'Académie celtique et George Sand. Les débuts des recherches folkloriques en France », Romantisme, no 9 : 29-38.

Belmont, Nicole. 1986. « Le folklore refoulé ou les séductions de l'archaïsme », L'Homme, no $97-98: 259-268$.

Belmont, Nicole (dir.). 1995. Aux sources de l'ethnologie française. L'académie celtique. Paris, Éd. du Comité des travaux historiques et scientifiques, 1995.

Bénichou, Paul. 1970. Nerval et la chanson folklorique. Paris, José Corti.

Benini, Romain. 2014. "Chansons dites "populaires” imprimées à Paris entre 1848 et 1851, approche stylistique et métrique », thèse de doctorat en langue française (stylistique), École normale supérieure de Lyon.

Berthou-Bécam, Laurence et Didier Bécam. 2010. L'enquête Fortoul (1852-1876). Chansons populaires de Haute et Basse-Bretagne. Paris, Éd. du Comité des travaux historiques et scientifiques.

Blanckaert, Claude (dir.). 1996. Le terrain des sciences humaines : instructions et enquêtes, xviiie-xxe siècle. Paris et Montréal, L'Harmattan.

Bourdieu, Pierre. 1986. "L'illusion biographique », Actes de la recherche en sciences sociales, no 62-63: 69-72.

Campos, Rémy. 2013. « De la prise de note à l'analyse des musiques populaires sous la Troisième République », in Esteban Buch, Nicolas Donin et Laurent Feneyrou (dir.), Du politique en analyse musicale. Paris, Vrin : 87-102.

Certeau, Michel de, Dominique Julia et Jacques Revel. 1993 [1974]. «La beauté du mort », in Michel de Certeau, La culture au pluriel. Paris, Seuil : 45-72.

Certeau, Michel de, Dominique Julia et Jacques Revel. 2002 [1975]. Une politique de la langue. La Révolution française et les patois : l'enquête de Grégoire. Paris, Gallimard. 
Chaline, Jean-Pierre. 1998. Sociabilité et érudition. Les sociétés savantes en France, XIXeXXe siècles. Paris, Éd. du Comité des travaux historiques et scientifiques.

Charle, Christophe. 1979. La crise littéraire à l'époque du naturalisme, roman, théâtre et politique : essai d'histoire sociale des groupes et des genres littéraires. Paris, Presses de l'École normale supérieure.

Charles-Dominique, Luc. 2009. «L'apport de l'histoire de l'ethnomusicologie de la France », in Luc Charles-Dominique et Yves Defrance (dir.), L'ethnomusicologie de la France : de l'«ancienne civilisation paysanne » à la globalisation, actes du colloque «L'ethnomusicologie de la France », 15-18 novembre 2006. Paris, L'Harmattan : 119-154.

Charles-Dominique, Luc. 2013. « La patrimonialisation des formes musicales et artistiques. Anthropologie d'une notion problématique », Ethnologies, vol. 35, no 1 : 75-101.

Cheyronnaud, Jacques. 1986. Mémoires en recueils : jalons pour une histoire des collectes musicales en terrains français. Montpellier, Office départemental d'action culturelle.

Cheyronnaud, Jacques. 1997. Instructions pour un recueil général des poésies populaires de la France (1852-1857). Paris, Éd. du Comité des travaux historiques et scientifiques.

Christophe, Jacqueline, Denis-Michel Boell, Régis Meyran, Michel Colardelle, Isac Chiva et Christian Bromberger. 2009. Du folklore à l'ethnologie. Paris, Éd. de la Maison des sciences de l'homme.

Collini, Silvia et Antonella Vannoni (dir.). 2005. Les instructions scientifiques pour les voyageurs (XVIIe-XIXe siècle). Paris, Budapest et Turin, L’Harmattan.

Comité de la langue, de l'histoire et des arts de la France. 1854. Bulletin du Comité de la langue, de l'histoire et des arts de la France (1852-1853). Paris, Impr. impériale.

Condemi, Concetta. 1992. Les cafés-concerts. Histoire d'un divertissement, 1849-1914. Paris, Quai Voltaire.

Darriulat, Philippe. 2010. La muse du peuple : chansons politiques et sociales en France 1815-1871. Rennes, Presses universitaires de Rennes.

Dubuc, André. 1952. «L'enquête de 1853 sur la chanson populaire en Normandie », Annales de Normandie, vol. 2, no $2: 151-157$.

Eyerman, Ron et Andrew Jamison. 1998. Music and Social Movements: Mobilizing Traditions in the Twentieth Century. Cambridge et New York, Cambridge University Press.

Fabre, Daniel et Jean-Marie Privat (dir.). 2011. Savoirs romantiques : une naissance de l'ethnologie. Nancy, Presses universitaires de Nancy.

Fortoul, Hippolyte. 1989. Journal d'Hippolyte Fortoul, Ministre de l'instruction publique et des cultes (1811-1856), éd. par Geneviève Massa-Gille. Genève, Droz.

Fulcher, Jane F. 1980. «The Popular Chanson of the Second Empire: "Music of the Peasants" in France », Acta Musicologica, vol. 52, fasc. 1 : 27-37.

Gaidoz, Henri. 1906. « De l'étude des traditions populaires, ou folk-lore, en France et à l'étranger », Bulletin de la Société Ramond, t. 1, nos 3-4 : 174-193.

Gauthier, Claudine. 2013. « Histoires croisées. Folklore et philologie de 1870 à 1920 », Les carnets de Bérose, no 1.

Gelbart, Matthew. 2007. The Invention of «Folk Music » and «Art Music »: Emerging Categories from Ossian to Wagner. Cambridge et New York, Cambridge University Press (New perspectives in music history and criticism).

Gellner, Ernest. 1983. Nations and Nationalism. Oxford, Blackwell. 
Gerson, Stéphane. 2003. The Pride of Place: Local Memories and Political Culture in Nineteenth-Century France. Ithaca et Londres, Cornell University Press.

Goody, Jack. 1986 [1978]. La raison graphique. La domestication de la pensée sauvage, trad. par Jean Bazin et Alban Bensa. Paris, Éd. de Minuit.

Gribaudi, Maurizio et Michèle Riot-Sarcey. 2009. 1848 : la révolution oubliée. Paris, La Découverte.

Grignon, Claude et Jean-Claude Passeron. 2015 [1989]. Le savant et le populaire : misérabilisme et populisme en sociologie et en littérature. Paris, Points.

Guillaumin, Colette. 2002 [1972]. L’idéologie raciste : genèse et langage actuel. Paris, Gallimard.

Hayat, Samuel. 2014. Quand la République était révolutionnaire : citoyenneté et représentation en 1848. Paris, Seuil.

Higonnet, Patrice L.-R. 1980. « The Politics of Linguistic Terrorism and Grammatical Hegemony during the French Revolution », Social History, vol. 5, no 1 : 41-69.

Hobsbawm, Eric John et Terence Osborn Ranger (dir.). 1983. The Invention of Tradition. Cambridge, Cambridge University Press.

Jeanpierre, Laurent. 2010. « Frontière », in Olivier Christin (dir.), Dictionnaire des concepts nomades en sciences humaines, t. 1. Paris, Métailié : 157-169.

Kimminich, Eva. 1991. "Chansons étouffées. Recherche sur les cafés concerts au XIXe siècle », Politix, no $14: 19-26$.

Kimminich, Eva. 1999. «Reformuler la réalité sociale en chantant », Regards sociologiques, nos 17-18: 71-84.

Laborde, Denis. 1998. Musiques à l'école. Paris, Bertrand-Lacoste.

Laferté, Gilles, Paul Pasquali et Nicolas Renahy. 2018. Le laboratoire des sciences sociales : histoires d'enquêtes et revisites. Paris, Raisons d'agir.

Lamont, Michèle et Virág Molnár. 2002. « The Study of Boundaries in the Social Sciences », Annual Review of Sociology, vol. 28 : 167-195.

Lamont, Michèle, Sabrina Pendergrass et Mark Pachucki. 2015. «Symbolic Boundaries », in James Wright (dir.), International Encyclopedia of the Social and Behavorial Sciences. Oxford, Elsevier : 850-855.

Leroy, Rodolphe. 2001. « Le Comité des travaux historiques et scientifiques (1834-1914) : entre animation et contrôle du mouvement scientifique en France », thèse pour le diplôme d'archiviste paléographe, Paris, École des Chartes.

Leterrier, Sophie-Anne. 1999. «Musique populaire et musique savante au XIXe siècle. Du "peuple" au "public" », Revue d'histoire du XIXe siècle, no 19 : 89-103.

Leterrier, Sophie-Anne. 2005. Le mélomane et l'historien. Paris, Armand Colin.

Leterrier, Sophie-Anne. 2006. «L'archéologie musicale au XIXe siècle : constitution du lien entre musique et histoire », Revue d'histoire des sciences humaines, no 14 : 49-69.

Linz, Juan J. 1997 [1993]. « Construction étatique et construction nationale », Pôle Sud, no 7 : 5-26.

Löfgren, Orvar. 1989. « The Nationalization of Culture », Ethnologica Europaea, vol. 47, no $1: 5-23$.

Mabru, Lothaire. 2007. "Propos préliminaires à une archéologie de la notion de "musique traditionnelle" », ethnographiques.org [en ligne], no 12. 
Massa-Gille, Geneviève. 1995. « Hippolyte Fortoul », in Jean Tulard (dir.), Dictionnaire du Second Empire. Paris, Fayard : 533-534.

Mayaud, Isabelle. 2018. "Sciences de la musique sans frontières ? Contribution à une sociologie du processus de primitivisation », thèse de doctorat en sociologie, Université Paris 8 Vincennes - Saint-Denis.

Millot, Hélène, Nathalie Vincent-Munnia, Marie-Claude Schapira et Michèle Fontana (dir.). 2005. La poésie populaire en France au xixe siècle : théories, pratiques et réception. Tusson, Du Lérot.

Muel-Dreyfus, Francine. 1983. Le métier d'éducateur. Les instituteurs de 1900, les éducateurs spécialisés de 1968. Paris, Éd. de Minuit.

Müller, Bertrand et Florence Weber. 2003. "Réseaux de correspondants et missions folkloriques. Le travail d'enquête, en France, vers 1930 », Gradhiva. Revue d'anthropologie et d'histoire des arts, no $33: 43-55$.

Niel, Frédérique. 2007. «Les vicissitudes de l'État linguistique (ou comment les langues minoritaires deviennent l'objet d'une politique sociale linguistique) : contribution à une sociologie historique du capital informationnel d'État ", thèse de doctorat en science politique, Université de Picardie- Jules-Verne.

Ponton, Rémy. 1977. «Les images de la paysannerie dans le roman rural à la fin du XIXe siècle », Actes de la recherche en sciences sociales, no $17: 62-71$.

Poulot, Dominique et Richard Wrigley. 1988. " The Birth of Heritage: "le moment Guizot" », Oxford Art Journal, vol. 11, no 2 : 40-56.

Pudal, Bernard. 1991. «Le populaire à l'encan », Politix, no 14 : 53-64.

Raphael, Paul et Maurice Gontard. 1975. Hippolyte Fortoul : 1851-1856. Paris, Puf.

Ravaisson, Félix. 1856. Funérailles de M. Fortoul le samedi 12 juillet 1856. Paris, Institut impérial de France, Académie des inscriptions et belles-lettres.

Simonsen, Michèle. 2005. «Poésies populaires de la France. The Fortoul Collection of French Folk Songs (1853-1855) », Estudos de literatura oral, nos 11-12 : 253-268.

Theis, Laurent. 1986. « Guizot et les institutions de mémoire », in Pierre Nora (dir.), Les lieux de mémoire, t. II : La Nation. Paris, Gallimard : 569-592.

Thiesse, Anne-Marie. 1997. Ils apprenaient la France. L'exaltation des régions dans le discours patriotique. Paris, Éd. de la Maison des sciences de l'homme.

Thiesse, Anne-Marie. 2001 [1999]. La création des identités nationales. Europe, xviiie-xxe siècle. Paris, Seuil.

Tiersot, Julien. 1931. La chanson populaire et les écrivains romantiques. Avec 96 notations musicales. Paris, Plon.

Traïni, Christophe. 2008. La musique en colère. Paris, Presses de Sciences Po.

Vaisse, Pierre. 2000. « Hippolyte Fortoul », Revue germanique internationale, no 13 : 141155.

Valière, Michel. 2002. Ethnographie de la France. Histoire et enjeux contemporains des approches du patrimoine ethnologique. Paris, Armand Colin (Cursus).

Vigier, Philippe. 1979. «Diffusion d'une langue nationale et résistance des patois en France au XIXe siècle », Romantisme, no 25-26 : 191-208.

Weber, Florence. 2000. « Le folklore, l'histoire et l'État en France (1937-1945) », Revue de synthèse, vol. 121, no 3-4 : 453-467. 
Weber, Max. 2002 [1959]. Le savant et le politique, trad. de l'allemand par Julien Freund. Paris, 10-18.

Weber, William. 1989. "Mentalité, tradition et origines du canon musical en France et en Angleterre au XVIIIe siècle », Annales. Économies, sociétés, civilisations, 44e année, no 4 : 849-873.

Weber, William. 1996. The Rise of Musical Classics in Eighteenth-Century England : A Study in Canon, Ritual, and Ideology. Oxford, Clarendon Press.

Yon, Jean-Claude. 2012 [2004]. Le Second Empire. Politique, société, culture. Paris, Armand Colin. 